\title{
Recurrence phenomenon for Vlasov-Poisson simulations on regular finite element mesh
}

\author{
M. Mehrenberger ${ }^{1,3}$, L. Navoret ${ }^{2,3}$, N. Pham ${ }^{2,3}$
}

November 27, 2018

\begin{abstract}
1. Institut de Mathématiques de Marseille,
Centre de Mathématiques et Informatique, UMR 7373, 39 rue Frédéric Joliot Curie, 13453 Marseille Cedex 13 France.
\end{abstract}

2. Institut de Recherche Mathématique Avancée, UMR 7501, Université de Strasbourg et CNRS, 7 rue René Descartes, 67000 Strasbourg, France

3. INRIA Nancy-Grand Est, TONUS Project, Strasbourg, France

\begin{abstract}
In this paper, we focus on one difficulty arising in the numerical simulation of the Vlasov-Poisson system: when using a regular grid-based solver with periodic boundary conditions, perturbations present at the initial time artificially reappear at a later time. For regular finite-element mesh in velocity, we show that this recurrence time is actually linked to the spectral accuracy of the velocity quadrature when computing the charge density. In particular, choosing trigonometric quadrature weights optimally defers the occurence of the recurrence phenomenon. Numerical results using the SemiLagrangian Discontinuous Galerkin and the Finite Element / Semi-Lagrangian method confirm the analysis.
\end{abstract}

\section{Keywords:}

\section{Introduction}

Accurate long run simulations of plasmas (gas of charged particles) are crucial in many applications like energy production. In this work, we are mainly interested in collisionless plasmas, whose dynamics can be described by a Vlasov-Poisson system under some suitable physical assumptions. The Vlasov equation is a transport equation satisfied by the kinetic distribution function of the particles in phase-space (position/velocity). Developing efficient and accurate numerical schemes remains a real challenge. Among several difficulties, one of them appears when solving the kinetic transport equations on 
a regular phase-space grid with periodic boundary conditions: a recurrence phenomenon occurs and suddenly destroys the accuracy of the numerical solution. In this paper, we show that the recurrence time is linked to the precision of the velocity quadrature when computing the charge density. In particular, choosing trigonometric quadrature weights optimally weaken the recurrence phenomenon.

The recurrence phenomenon is for the first time mentioned in the seminal paper of Cheng and Knorr [7], where they consider a regular grid of the phase space and a semi-Lagrangian scheme to solve the Vlasov equation. When considering periodic boundary conditions, the Vlasov dynamics exhibit phase mixing: small initial spatial perturbations result in oscillations in the velocity distribution, whose frequency grows in time. Consequently, at some time, the velocity mesh cannot capture these oscillations anymore. This leads to an aliasing effect: when charge density is computed, the large frequency oscillations are numerically interpreted as small frequency ones. Note that charge density and the electric field are related through the Poisson equation and thus the recurrence phenomenon is rather observed on the electric energy time evolution. When using a uniform grid, the recurrence time can be explicitly given in this simple case: $T=2 \pi /(k \Delta v)$, where $k$ is the spatial perturbation mode and $\Delta v$ is the velocity mesh size.

Vlasov solver based on a phase grid enable to reach high order accuracy. This is in contrast with particle methods [21], which do not involve velocity grid but are subject to statistical noise. The semi-Lagrangian method, used in [7], is a grid-based transport solver: each time step consists in computing the characteristics of the transport equation backward and interpolating the nodal values of the distribution function at the feet of the characteristics. Numerous variants of the methods have been studied, considering different interpolation operators (e.g. spline, Lagrange, Hermite interpolation), conservative version of the method. We refer to [28] and references therein. Their main advantage is to overcome the CFL stability condition. Another class of grid-based methods are the Eulerian one, like finite-volume [17] or discontinuous Galerkin method [5,19]. These methods handle more naturally complex geometries at the price of satisfying the CFL condition.

High-order accurate grid-based solver are designed to carry out long-run simulations and recurrence phenomenon is a real limitation. The simplest way to extend the recurrence time is to refine the mesh by taking a smaller $\Delta v$. However, it often demands too much computation time and storage ressources. In consequence, several works have considered methods to suppress or at least reduce the recurrence phenomenon. The main method consists in using filtering techniques. This can be done by adding an artificial diffusion to the Vlasov equation [22], adding outflow boundary conditions in the Fourier velocity variable [14-16] or using cutoff procedure in Fourier space [13]. For the latter method, the influence of the cutoff on the invariants of the dynamics, such as mass, entropy or $L^{p}$ norms, has been studied in [11]. Filtering techniques have also been studied and analysed in the context of the Hermite-spectral method [6]. In another direction, randomization of the velocity points in each velocity cells have been considered in [1].

In this work, we focus on methods based on a finite-element mesh in velocity, like the discontinuous-Galerkin method or the semi-Lagrangian discontinous-Galerkin (SLDG) 
scheme. Each time step of these methods consists in advecting a piecewise polynomial reconstruction of the distribution function and then take its $L^{2}$ projection on the discrete functionnal space. These methods have the advantage to be $L^{2}$ stable by construction. The SLDG method has been introduced in $[10,26,27]$ and analysed in [12]. The discontinuous Galerkin method have been introduced and analysed for the Vlasov equation in $[5,19]$. Another method based on reduced Vlasov equation, obtained from a finite-element discretization in velocity, has been introduced in $[18,20]$ : it can possibly be implemented in any hyperbolic code.

When using a uniform finite-element mesh in velocity, it has been observed surprisingly that the recurrence phenomenon behaves unfavourably when increasing the degree of approximation $[8,23,25]$, see also [11] for the plasma echo phenomenon. Let consider a finite-element mesh of degree $d$, which implies that there are $d+1$ degrees of freedom in each velocity cell. The goal of this paper is to formally show that the recurrence time only depends a priori on the mesh size $\Delta v$ and not on the degree: $T_{\text {rec }}=2 \pi /(k \Delta v)$. More precisely, computing the charge density requires the choice of a quadrature rule and the amplitude of the recurrence phenomenon is related to the precision of this method on the trigonometric polynomials. From our knowledge, it is the first work in this direction. Note that this analysis is performed on the kinetic transport equation and not on the full Vlasov-Poisson system. However, we show numerically that the results are still valid in that case. The Vlasov equation is solved using the SLDG scheme. We carry out also some numerical simulations using the Finite Element/Finite Volume method (FE/FV) [20].

From this formal analysis, a natural idea to extend the time before recurrence is to use trigonometric quadrature weights when computing the charge density. Indeed, the error on trigonometric polynomials up to order $\lfloor d / 2\rfloor$ then vanishes and the recurrence time equals: $T_{\mathrm{rec}}^{\mathrm{trigo}}=2 \pi(\lfloor d / 2\rfloor+1) /(k \Delta v)$. The properties of this quadrature rule has been recently studied in $[2,3]$. Note that the trigonometric quadrature is only used in the computation of the density.

This provides a simple method to modify existing codes and potentially delay the recurrence time. In the numerical simulations, we will see that the benefit of the method is however restricted to low degree of approximation and linearized regimes. The analysis yet quantify the recurrence phenomenon and also provide properties that can help to certificate codes.

The plan of the paper is the following. In Section 2, we perform the analysis of the recurrence phenomenon on the transport equation. In Section 3, we introduce the method based on the trigonometric quadrature rules. Finally, numerical simulations on the Vlasov-Poisson system are carried out to show that the previous study is still valid in this case. Three appendices complete this work: the first one provides the derivation of the trigonometric rules and the two other present the SLDG and the FE/FV scheme. 


\section{Recurrence on transport equation}

Let consider a kinetic distribution $f(x, v, t)$ in the two dimensional phase space, $(x, v) \in$ $\Omega_{x} \times \Omega_{v} \subset \mathbb{R}^{2}$, and $t \in \mathbb{R}^{+}$. We consider the one-dimensional transport equation:

$$
\partial_{t} f+v \partial_{x} f=0, \quad \forall(x, v, t) \in \Omega_{x} \times \Omega_{v} \times \mathbb{R}_{+} .
$$

on the periodic domain $\Omega_{x}=[0,2 \pi / k]$ and $\left.\Omega_{v}=\right] v_{\min }, v_{\max }\left[\right.$ with $v_{\min }=-v_{\max }$. Considering the following initial data:

$$
f_{0}(x, v)=\cos (k x) M(v),
$$

with $k \in \mathbb{N}$ and $M(v)=\exp \left(-v^{2} / 2\right) / \sqrt{2 \pi}$ denoting the Gaussian function. The exact solution is given by:

$$
f(x, v, t)=\cos (k(x-v t)) M(v) .
$$

The solution exhibits filamentation in phase-space: an initial $k$-th spatial mode leads to a $k t$ mode in velocity at time $t>0$.

\section{$2.1 \quad$ Finite element mesh}

We first introduce the finite element mesh. We consider a uniform mesh of the domain $\Omega_{v}$ with step size $\Delta v=\left(v_{\max }-v_{\min }\right) / N_{v}$, with $N_{v} \in \mathbb{N}^{*}: N_{v}$ is the total number of mesh cells. The mesh points are given by:

$$
v_{j}=j \Delta v, \quad \forall j \in \llbracket-N_{v} / 2, N_{v} / 2 \rrbracket,
$$

Then, given an approximation degree $d \in \mathbb{N}$, we introduce in each cell $d+1$ points. Let $\left(\bar{v}_{\ell}\right)_{0 \leqslant \ell \leqslant d}$ denote points in the interval $[0,1]$. For instance, they can be uniform or be Gauss-Legendre or Gauss-Lobatto points to further obtain optimal integration accuracy. We thus consider the discretization points

$$
v_{(j, \ell)}=v_{j}+\Delta v \bar{v}_{\ell}, \quad \forall(j, \ell) \in K:=\llbracket-N_{v} / 2, N_{v} / 2-1 \rrbracket \times \llbracket 0, d \rrbracket,
$$

Note that, if the boundary points are included $\left(\min \bar{v}_{\ell}=0\right.$ and $\left.\max \bar{v}_{\ell}=1\right)$, the mesh points contains redundancies.

We further suppose that the mesh points are symmetrically distributed with respect to 0 . In particular, we have:

$$
\forall(j, \ell) \in K, \quad v_{-j, d-\ell}=-v_{j-1, \ell} .
$$

This symmetry holds if and only if the points $\left(\bar{v}_{\ell}\right)_{0 \leqslant \ell \leqslant d}$ are symmetrically distributed. This is in particular true for Gauss points.

We then consider quadrature weights $\left(\omega_{\ell}\right)_{0 \leqslant \ell \leqslant d}$ associated to $\left(\bar{v}_{\ell}\right)_{0 \leqslant \ell \leqslant d}$. The aim of the paper is to determine condition on quadrature weights to weaken the recurrence phenomenon. 


\subsection{Semi-discrete solution in velocity}

We consider the following set of transport equations:

$$
\partial_{t} f_{\mathrm{sd},(j, \ell)}+v_{(j, \ell)} \partial_{x} f_{\mathrm{sd},(j, \ell)}=0, \quad \forall(j, \ell) \in K .
$$

The functions $\left(f_{\mathrm{sd},(j, \ell)}(x, t)\right)$ are a nodal semi-discretization of the distribution function in velocity. Index "sd" refers to "semi-discrete". We consider the following initial condition:

$$
f_{\mathrm{sd},(j, \ell)}(x, t=0)=\cos (k x) M\left(v_{(j, \ell)}\right), \quad \forall(j, \ell) \in K .
$$

The exact solution to these independent transport equations is thus given by:

$$
f_{\mathrm{sd},(j, \ell)}(x, t)=\cos \left(k\left(x-v_{(j, \ell)} t\right)\right) M\left(v_{(j, \ell)}\right), \quad \forall(j, \ell) \in K .
$$

In the special case where $d=0$, the mesh is uniform and all these functions hare periodic with the same period $2 \pi /(k \Delta v)$ which leads to the recurrence: the initial solution reappear at time $T_{\text {rec }}=2 \pi /(k \Delta v)$. In the general case, these functions have no more a common period and this argument no more valid. Nevertheless, we will see in the next section that this recurrence time is still relevant.

This nodal semi-discretization in velocity underlies different numerical schemes like finite difference or the semi-Lagrangian schemes. The latter is now a standard method for solving kinetic equations in plasma physics. Continuous or discontinuous Galerkin method does not enter directly the framework since the transport systems is usually not diagonal. However, we still expect the same kind of behavior.

\subsection{Time correlation of the density and recurrence}

To analyze time correlation of solution (5), we consider the following quantity:

$$
C_{\mathrm{sd}}(t)=\int_{\Omega_{x}} \rho_{\mathrm{sd}}(x, 0) \rho_{\mathrm{sd}}(x, t) d x .
$$

where $\rho_{\text {sd }}(x, t)$ denotes the approximate density associated to the semi-discrete distribution function $\left(f_{\mathrm{sd},(j, \ell)}(x, t)\right)$. Using the quadrature rules to integrate the distribution function (5) in velocity, this approximate density writes:

$$
\rho_{\mathrm{sd}}(x, t)=\sum_{(j, \ell) \in K} \Delta v \omega_{\ell} f_{\mathrm{sd},(j, \ell)}(x, t)=\Delta v \sum_{(j, \ell) \in K} \omega_{\ell} \cos \left(k\left(x-v_{(j, \ell)} t\right)\right) M\left(v_{(j, \ell)}\right) .
$$

Using the symmetry of the discrete velocities distribution and oddness of the sine function, density simplifies into:

$$
\rho_{\mathrm{sd}}(x, t)=\cos (k x) h(t),
$$

where function $h(t)$ is defined by:

$$
h(t)=\Delta v \sum_{(j, \ell) \in K} \omega_{\ell} \cos \left(k v_{(j, \ell)} t\right) M\left(v_{(j, \ell)}\right) .
$$


Correlation function (6) is thus given by:

$$
C_{\text {sd }}(t)=\frac{\pi}{k} h(t) h(0)
$$

We then perform a rescaling in time $t=\tilde{t}(2 \pi) /(k \Delta v)$ and introduce $\tilde{h}(\tilde{t})=h(t)$ and $\tilde{C}_{\text {sd }}(\tilde{t})=C_{\text {sd }}(t)$. Using the expression of the discrete velocities (2), we get:

$$
\tilde{h}(\tilde{t})=\Delta v \sum_{(j, \ell) \in K} \omega_{\ell} \cos \left(2 \pi\left(j+\bar{v}_{\ell}\right) \tilde{t}\right) M\left(v_{(j, \ell)}\right) .
$$

A straigthforward computation shows that integer times $\tilde{t} \in \mathbb{N}$ are local extrema of this function (still using the symmetry of the points). For any $m \in \mathbb{N}$, we have:

$$
\begin{aligned}
\tilde{h}(m) & =\sum_{\ell=0}^{d} \omega_{\ell} \cos \left(2 \pi m \bar{v}_{\ell}\right)\left[\sum_{j=-N_{v} / 2}^{N_{v} / 2-1} \Delta v M\left(v_{(j, \ell)}\right)\right] \\
& =\beta\left(\sum_{\ell=0}^{d} \omega_{\ell} \cos \left(2 \pi m \bar{v}_{\ell}\right)\right)+O(\Delta v),
\end{aligned}
$$

where $\beta$ is a constant given by: $\beta=\int_{v_{\min }}^{v_{\max }} M(v) d v$. Finally, using (8) and (9), correlation function has the following expression:

$$
\forall m \in \mathbb{N}, \quad \tilde{C}_{\text {sd }}(m)=\frac{\pi}{k} \beta^{2}\left(\sum_{\ell=0}^{d} \omega_{\ell} \cos \left(2 \pi \bar{v}_{\ell} m\right)\right)+O(\Delta v) .
$$

Consequently, the correlation at time $m(2 \pi) /(k \Delta v)$, with $m \in \mathbb{N}^{*}$, is quantified by the numerical error of the quadrature rule on the trigonometric function $x \mapsto \cos (2 \pi m)$ (since $\left.\left.\int_{0}^{1} \cos (2 \pi v \tilde{t}) d v=0\right)\right)$. Note that this correlation does not vanish as $\Delta v$ goes to 0 .

The correlation of the exact solution (1) have an exponential decrease in time. Indeed, supposing that the velocity domain is infinite $\Omega_{v}=\mathbb{R}$, the density has the following explicit expression:

$$
\rho(x, t)=\cos (k x) \sqrt{2 \pi} e^{-\frac{k^{2} t^{2}}{2}},
$$

and the correlation function equals:

$$
C(t)=\int_{\Omega_{x}} \rho(x, 0) \rho(x, t) d x=\frac{2 \pi^{2}}{k} e^{-k^{2} t^{2}} .
$$

In particular, for any $m \in \mathbb{N}^{*}$, we have: $\tilde{C}(m):=C(m(2 \pi) /(k \Delta v))=\frac{2 \pi^{2}}{k} e^{-(2 \pi)^{2}(m / \Delta v)^{2}}$ that tends to 0 as $\Delta v \rightarrow 0$, which is not the case for the semi-discrete correlation function (10).

Without additional assumptions on the quadrature rule, the recurrence phenomenon may hold at time $\tilde{t}_{\text {rec }}=1$ that is:

$$
T_{\mathrm{rec}}=\frac{2 \pi}{k \Delta v}
$$



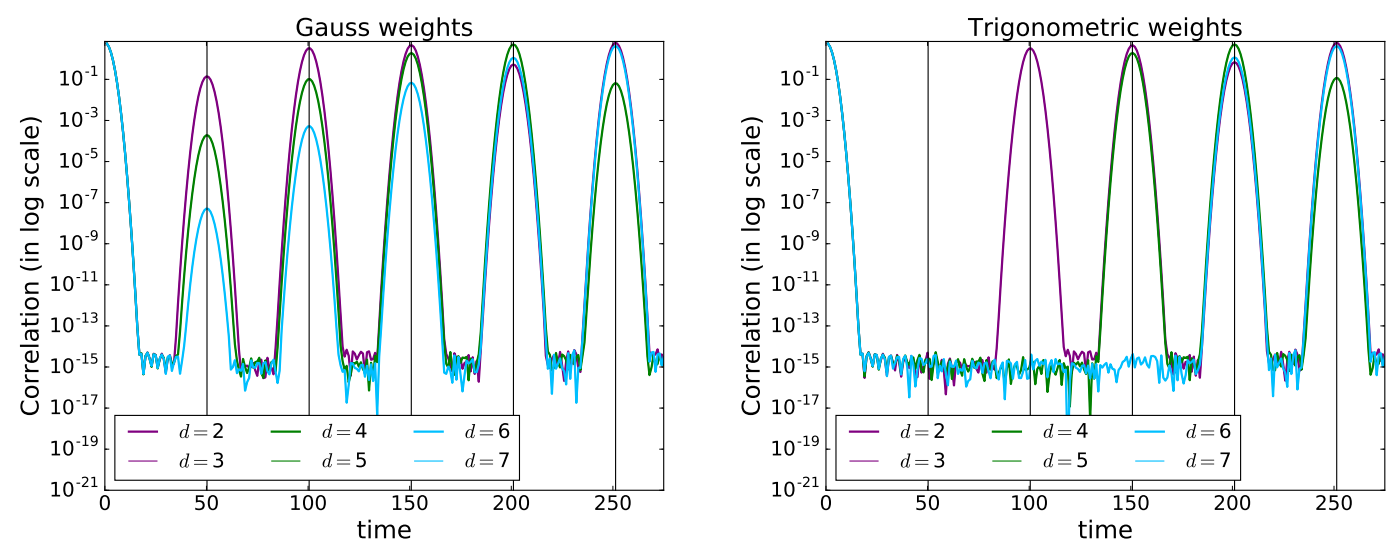

Figure 1: (Free transport equation) Absolute value of the correlation $C_{\mathrm{sd}}(t)$ as a function of time using Gauss-Legendre points for degree $2 \leqslant d \leqslant 7$. Parameters: $N_{v}=64$ and $k=0.5$. The black vertical lines indicates the multiple of the recurrence time $T_{\text {rec }}=2 \pi /(k \Delta v) \approx 50.26$. Left: Gauss weights. Right: Trigonometric weights.

The first recurrence time thus does not depend on approximation degree $d$ and thus on the accuracy of the scheme. However, we note that the amplitude of the correlation depends on the precision of the quadrature rule. In Figure 1 (left), we plotted the correlation $C_{\mathrm{sd}}(t)$ as a function of time using Gauss-Legendre points and weights. We actually see that the correlation is very small at time $T_{\text {rec }}=\frac{2 \pi}{k \Delta v}$ as soon as the degree $d$ is larger than 3 , but it is actually non zero. We will discuss this in more details in the next section.

In the specific case of uniform velocity points $\left(v_{\ell}=(2 \ell+1) /(2 d+2)\right)$ and uniform weights $\left(\omega_{\ell}=1 /(d+1)\right)$, the quadrature rule is actually exact on trigonometric polynomials of degree up to $d$. Hence, the order $O(1)$ term of the correlation vanishes for $\tilde{t} \leqslant d$ and the recurrence phenomenon may occur only for $\tilde{t}_{\text {rec }} \geqslant d+1$ that is $T_{\text {rec }}=(d+1)(2 \pi /(k \Delta v))$ : the recurrence time involves the finer periodic scale of the mesh, here $\Delta v /(d+1)$.

\section{Trigonometric quadrature rules and recurrence}

Given expression (10) and the previous discussion, ensuring that the recurrence time is as large as possible leads to consider quadrature rules that are exact on trigonometric polynomials. These are called trigonometric quadrature rules. Theoretical condition ensuring the positivity of the weights has been given in [24].

Given $d+1$ velocity points $\left(\bar{v}_{\ell}\right)_{0 \leqslant \ell \leqslant d}$ in the interval $[0,1]$, we can determine integration weights ensuring the exact integration of trigonometric polynomials of degree lower or 
equal to $\lfloor d / 2\rfloor$ :

$$
\int_{0}^{1} e_{m}(v) d v=\sum_{\ell=0}^{d} \omega_{\ell} e_{m}\left(v_{\ell}\right), \quad \forall m \in \llbracket-\lfloor d / 2\rfloor,\lfloor d / 2\rfloor \rrbracket,
$$

with $e_{m}(v)=e^{2 i \pi m v}$. Weights can be computed explicitly using the following formula (see appendix A and [2]):

$$
\forall \ell \in \llbracket 0,\lfloor d / 2\rfloor \rrbracket, \quad \omega_{\ell}=\omega_{d-\ell}=C_{\ell} \int_{0}^{1}\left(\prod_{\substack{0 \leqslant j \leqslant\lfloor d / 2\rfloor \\ j \neq \ell}} \frac{\cos (2 \pi v)-\cos \left(2 \pi \bar{v}_{j}\right)}{\cos \left(2 \pi \bar{v}_{\ell}\right)-\cos \left(2 \pi \bar{v}_{j}\right)}\right) d v .
$$

with $C_{\ell}=1 / 2$ if $\ell \neq d / 2$ and $C_{\ell}=1$ if $\ell=d / 2$ (even $\left.d\right)$.

With this trigonometric rule, the order $O(1)$ term of the correlation vanishes for $\tilde{t} \leqslant\lfloor d / 2\rfloor$ and the recurrence phenomenon occurs only for $\tilde{t}_{\text {rec }} \geqslant\lfloor d / 2\rfloor+1$ that is:

$$
T_{\text {rec }}^{\text {trigo }}=(\lfloor d / 2\rfloor+1) \frac{2 \pi}{k \Delta v} .
$$

In Figure 1 (right), we plotted the correlation $C_{\text {sd }}(t)$ as a function of time using GaussLegendre points and trigonometric weights. We actually observe that the first local maximum of the correlation function occurs at this recurrence time.

Gauss-Legendre points. Let consider the Gauss-Legendre velocity points (rescaled on the interval $[0,1)$ ). In Table 1 , we report the numerical errors when integrating trigonometric polynomials of degree $m \leqslant d$ using the Gauss-Legendre weights or the trigonometric weights. We see that the Gauss-Legendre do not exactly integrate trigonometric polynomials of degree less than $\lfloor d / 2\rfloor$. However, being of optimal order $\left(O\left(\Delta v^{2 d+1}\right)\right.$ for $d+1$ points $)$, the results are still quite satisfactory: for instance, the error for $m=0$ equals $8.3 \times 10^{-9}$ int he case $d=6$, which should result in small amplitude recurrence at time $t=2 \pi /(k \Delta v)$. We also mention that numerical computations shows that trigonometric weights (computed on Gauss-Legendre points) are always positive for $d \leqslant 18$. For larger $d>18$, weights are not all positive and thus the trigonometric quadrature should be avoided.

Uniform points. Note that for uniform points $\left(v_{\ell}=\ell /(d+1)\right)$, the integration weight are the inverse discrete Fourier transform (DFT) of $\hat{\omega} \in \mathbb{C}^{d+1}$, with $\hat{\omega}_{0}=0$ and $\hat{\omega}_{k}=1$ for $k \neq 0$. The weights are then all equal to $1 /(d+1)$ and we recover the trapezoidal rule. Moreover, in that case, all trigonometric polynomials up to degree $d$ are exactly integrated.

Gauss-Lobatto points. In Table 2, we have reported the numerical errors when using the Gauss-Lobatto points and the quadrature rules associated to Gauss weights or trigonometric weights. Like in the case of the Gauss-Legendre points, we observe 


\begin{tabular}{cccc}
$d$ & $m$ & GaussW & TrigoW \\
\hline \multirow{3}{*}{2} & 1 & 0.02245 & 0 \\
& 2 & 0.52996 & 0.51916 \\
& 3 & 0.73652 & 0.73047 \\
\hline & 1 & $1.0678 \times 10^{-3}$ & 0 \\
3 & 2 & 0.12573 & 0.12664 \\
& 3 & 0.74097 & 0.74155 \\
& 4 & 0.33811 & 0.33830 \\
\hline & 1 & $3.0803 \times 10^{-5}$ & 0 \\
& 2 & 0.016669 & 0 \\
4 & 3 & 0.30402 & 0.30657 \\
& 4 & 0.79870 & 0.80082 \\
& 5 & 0.010458 & 0.018835
\end{tabular}

\begin{tabular}{cccc}
$d$ & $m$ & GaussW & TrigoW \\
\hline \multirow{4}{*}{5} & 1 & $5.9704 \times 10^{-7}$ & 0 \\
& 2 & $1.4223 \times 10^{-3}$ & 0 \\
& 3 & 0.071038 & 0.073117 \\
& 4 & 0.50843 & 0.50769 \\
& 5 & 0.67454 & 0.67455 \\
& 6 & 0.30953 & 0.30852 \\
\hline & 1 & $8.3166 \times 10^{-9}$ & 0 \\
& 2 & $8.5037 \times 10^{-5}$ & 0 \\
& 3 & 0.010947 & 0 \\
6 & 4 & 0.17907 & 0.18741 \\
& 5 & 0.66873 & 0.66483 \\
& 6 & 0.40512 & 0.40575 \\
& 7 & 0.41364 & 0.41456
\end{tabular}

Table 1: (Gauss-Legendre points) Numerical quadrature errors on trigonometric polynomials of degree $m \leqslant d$ using Gauss-Legendre points and Gauss weights (GaussW) or trigonometric weights (TrigoW).

\begin{tabular}{|c|c|c|c|c|c|c|c|c|}
\hline$m$ & GaussW & TrigoW & $m$ & GaussW & TrigoW & $m$ & GaussW & TrigoW \\
\hline 1 & $1 / 3$ & 0 & 1 & $1.3174 \times 10^{-3}$ & 0 & 1 & $6.9354 \times 10^{-7}$ & 0 \\
\hline 2 & 1 & 1 & 2 & 0.14855 & 0 & 2 & $1.6300 \times 10^{-3}$ & 0 \\
\hline 3 & $1 / 3$ & 0 & 3 & 0.79651 & 0.93393 & 3 & 0.079357 & 0 \\
\hline \multirow[t]{5}{*}{4} & 1 & 1 & 4 & 0.25734 & 0.12776 & 4 & 0.542841 & 0.663454 \\
\hline & & & 5 & 0.10028 & 0.11932 & 5 & 0.64533 & 0.51383 \\
\hline & & & & & & 6 & 0.34435 & 0.22468 \\
\hline & & & & & & 7 & 0.15274 & $2.1373 \times 10^{-3}$ \\
\hline & $d=2$ & & & $d=4$ & & & $d=6$ & \\
\hline
\end{tabular}

Table 2: (Gauss-Lobatto points) Numerical quadrature errors on trigonometric polynomials of degree $m \leqslant d$ using Gauss-Lobatto points and Gauss weights (GaussW) or trigonometric weights (TrigoW).

that the Gauss weights lead to non zero but small errors on trigonometric polynomials lower than $\lfloor d / 2\rfloor$. We note that the error is larger than for the Gauss-Legendre method, due to the smaller degreee of accuracy $\left(O\left(\Delta v^{2 d-1}\right)\right.$ for $d+1$ points). The trigonometric quadrature is thus more attractive in the case of Gauss-Lobatto points. For the GaussLobatto points, the trigonometric weights are positive only up to $d=7$.

\section{Application to the Vlasov-Poisson dynamics}

In this section, we consider the Vlasov-Poisson dynamics still in the two-dimensional phase space: $(x, v) \in \Omega_{x} \times \Omega_{v} \subset \mathbb{R}^{2}$, and $t \in \mathbb{R}^{+}$. The kinetic distribution function 
$f(x, v, t)$ satisfies the following system:

$$
\begin{aligned}
& \partial_{t} f+v \partial_{x} f-E \partial_{v} f=0 \\
& E=-\partial_{x} \Phi, \quad-\partial_{x^{2}} \Phi=\rho_{0}-\rho(x, t) .
\end{aligned}
$$

where $E(x, t)$ is the self-consistent electric field, $\Phi(x, t)$ is the electric potential, $\rho(x, t)$ is the charge density:

$$
\rho(x, t)=\int_{\Omega_{v}} f(x, v, t) d v .
$$

and $\rho_{0}=\int_{\Omega_{x}} \rho(x, t=0) d x>0$. This electric field gives rise to a transport in the velocity direction. The recurrence phenomenon can be observed when looking at the electric energy:

$$
\mathcal{E}(t)=\int_{\Omega_{x}}(E(x, t))^{2} d x .
$$

Note that this quantity is also the $L^{2}$ norm of the primitive of the density.

Using classical test cases, we numerically study how the previous results extend to this non-linear transport system. To this aim, we consider two methods based on a finite element mesh (at least in velocity). The first method is the semi-Lagrangian discontinous Galerkin (SLDG) method $[10,26]$. The second one is the finite element/semi-Lagrangian (FE/SL) method $[18,20,25]$. For the sake of completeness, the two methods are described in appendices $\mathrm{B}$ and $\mathrm{C}$ respectively.

\subsection{Landau damping test cases}

The linear Landau damping test case consists in considering a small spatial perturbation of the space-homogeneous Maxwellian distribution in velocity. The initial distribution function is given by:

$$
f(0, x, v)=\frac{1}{\sqrt{2 \pi}} e^{-v^{2} / 2}(1+\alpha \cos (k x)),
$$

with $\alpha \ll 1$ and $k \in \mathbb{R}$. The computational domain is still given by $\Omega_{x}=[0,2 \pi / k]$ and $\left.\Omega_{v}=\right] v_{\min }, v_{\max }\left[\right.$ with $v_{\min }=-v_{\max }$. In the following simulation, we will take $k=0.5$.

Gauss versus trigonometric weights $\left(\alpha=10^{-6}\right)$. We first consider a perturbation size equal to $\alpha=10^{-6}$ and the SLDG method with Gauss-Legendre points. In Figure 2, we plot the time evolution of the electric energy $\mathcal{E}(t)$ (in log scale) when using the SLDG method for both Gauss weights and trigonometric weights. We use with $N_{v}=64, v_{\max }=$ 8 and different degree of approximation: $d=2, \ldots, 7$. As explained in the appendix (see ...), the choice of the quadrature weights only modifies the computation of the charge density $\rho$ : everything else in the algorithm is unchanged. We have also plotted vertical lines corresponding to the multiples of the recurrence time $T_{\text {rec }}=2 \pi /(k \Delta v) \approx 50.26$.

The theory of the linearized Vlasov-Poisson system predicts the damping of the electric energy to the rate -1.553 for the given wave number $k=0.5$ [28]. The sudden 

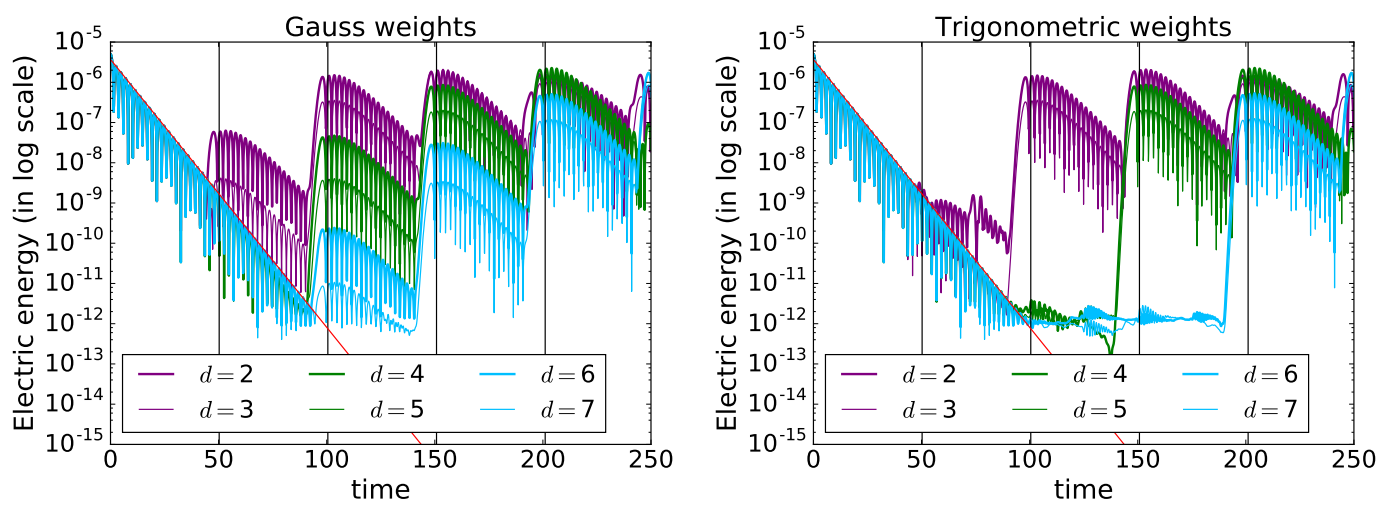

Figure 2: (Landau damping, SLDG, $k=0.5, \alpha=10^{-6}$ ) Time evolution of the electric energy $\mathcal{E}(t)$ (in log scale) when varying $d=2, \ldots, 7$. The black vertical lines indicates the multiple of the recurrence time $T_{\text {rec }}=2 \pi /(k \Delta v) \approx 50.26$. The red line is the theoretical damping of the linearized Vlasov-Poisson system. Parameters: $N_{x}=N_{v}=64, v_{\max }=8$, Gauss-Legendre points in space and velocity.

rises of the electric energy are due to the recurrence effect. We observe that they actually occur at multiple times of the recurrence time. Using trigonometric weights (right figure) enables the first large recurrence effect to be at time $T_{\text {rec }}^{\text {trigo }}=(\lfloor d / 2\rfloor+1) 2 \pi /(k \Delta v)$ as predicted by the analysis made in the previous section on the free transport equation. We yet observe that for $d=2$ the recurrence is only diminished and not totally shifted in time, as non-linear effects are involved.

Gauss versus trigonometric weights $\left(\alpha=10^{-2}\right)$. In Figure 3, we have plotted the results obtained when considering a larger perturbation amplitude, $\alpha=10^{-2}$. We here consider the FESL method, using a finite element method with Gauss-Lobatto points in velocity and a high-order semi-Lagrangian scheme in space (Lagrange interpolation, $d_{x}=$ $6)$. Gauss-Lobatto weights and trigonometric weights are compared. The recurrence is still reduced when using the trigonometric weights. It is particularly the case for $d=3$, for which we recover the right damping rate on a longer time. For $d=2$ and $d=4$, only the amplitude of the electric energy is reduced, but the damping dynamics is not resolved.

Gauss versus uniform points $\left(\alpha=10^{-6}\right)$. Since we noticed that uniform points results in large recurrence time when using trigonometric weights, we here consider the SLDG method, where the piece-wise polynomial approximation is expressed using uniform points $\left(\bar{v}_{j}=(j+0.5) /(d+1)\right.$ for $\left.j \in \llbracket 0, d \rrbracket\right)$ instead the standard Gauss-Legendre points. One advection step is thus identical to the SLDG scheme : this is only a change of the polynomial basis representation. Schemes yet differ when performing the splitting in advection and velocity as the reconstruction in each direction of the piece-wise polynomials depends on the considered points. 

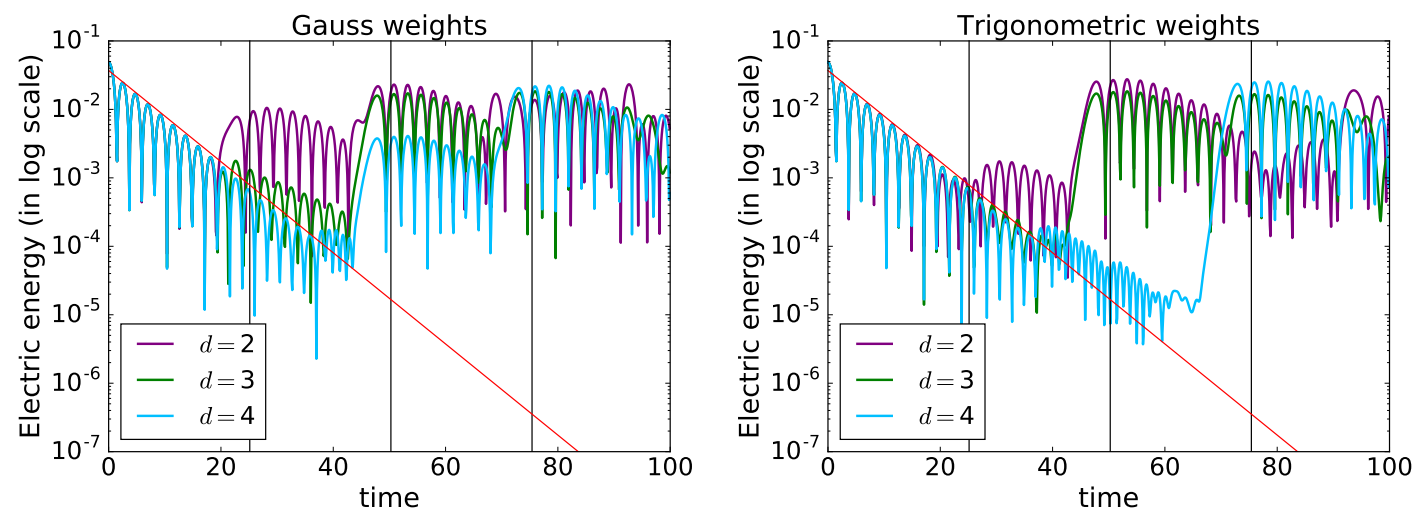

Figure 3: (Landau damping, FESL, $k=0.5, \alpha=10^{-2}$ ) Time evolution of the electric energy $\mathcal{E}(t)$ (in log scale) when varying $d=2,3,4$. The black vertical lines indicates the multiple of the recurrence time $T_{\text {rec }}=2 \pi /(k \Delta v) \approx 25.13$. Parameters: $N_{x}=N_{v}=16$, $v_{\max }=4, d_{x}=6$, Gauss-Lobatto points in velocity.

In Figure 4, we compare the results when using Gauss-Legendre or uniform points in velocity for degree $d=6$. We first observe that the amplitude of the electric energy at the first recurrence time is larger for uniform points than for Gauss-Legendre points, when using the classical quadrature rules (the Gauss weights for the Gauss points and the Newton-Côtes weights for the uniform points). Furthermore, as expected, considering trigonometric weights instead enables to shift the first significant recurrence at time $T_{\text {rec }}^{\text {trigo }}=(\lfloor 6 / 2\rfloor+1) 2 \pi /(k \Delta v)$ for the Gauss points and at time $T_{\text {rec }}^{\text {unif }}=(6+1) 2 \pi /(k \Delta v)$ for the uniform points.

Gauss versus uniform points $\left(\alpha=10^{-2}\right)$. In Figure 5, we make the same comparison as previously in the case where the perturbation has amplitude $\alpha=10^{-2}$. For degree $d=2$ (top figures), the same remarks still hold: uniform points leads to a larger first recurrence and trigonometric weights enable recurrence time to be larger. However the results are not as clear as previously due to the involved non-linear dynamics.

For larger degree of approximation, $d=6$ (bottom figures), it is even clearer that the use of uniform points deteriorates the solution from the recurrence point of view. Indeed, for $d=6$, the raise of electric energy at time $T_{\text {rec }}=2 \pi /(k \Delta v)$ is almost indistinguishable for Gauss points while it is significant for uniform points (bottom right). Trigonometric weights provide a remedy to this for uniform points, but the shift of the recurrence time is not as clean as for the case $d=2$. However, we still observe that the amplitude of the electric energy is lower for uniform points than for Gauss points up to time $t=175$. 

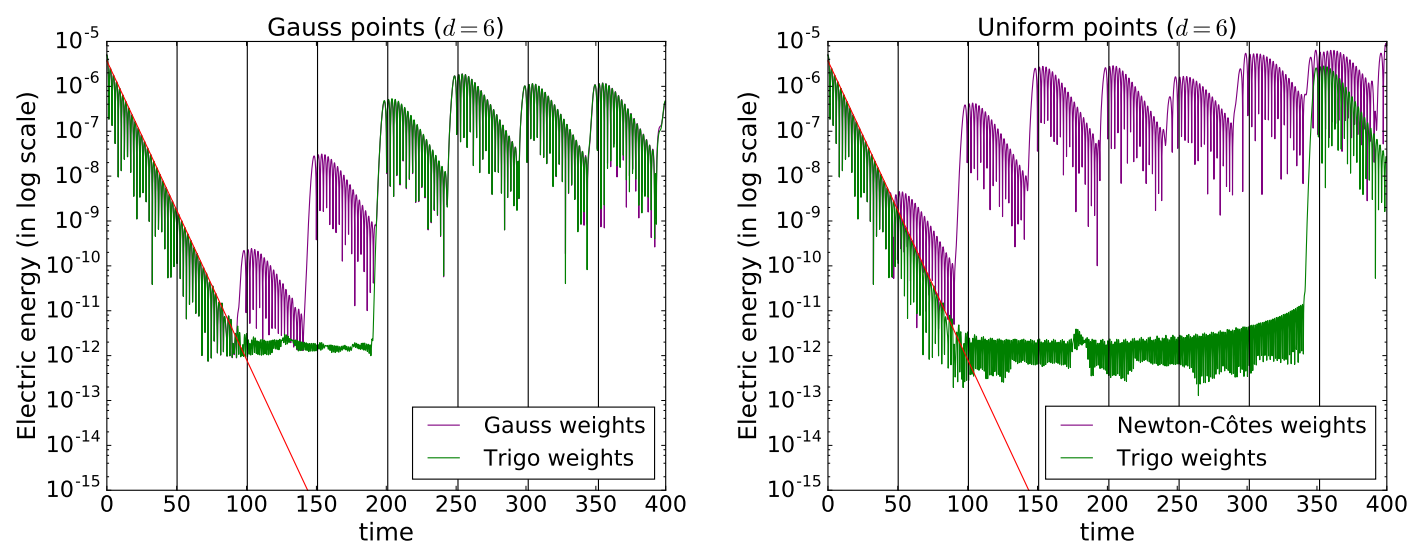

Figure 4: (Linear Landau damping, $\alpha=10^{-6}$ ) Time evolution of the electric energy $\mathcal{E}(t)$ (in $\log$ scale) when taking Gauss points (left) and uniform points (right). The black vertical lines indicates the multiple of the recurrence time $T_{\text {rec }}=2 \pi /(k \Delta v) \approx 50.26$. Parameters: $N_{v}=40, v_{\max }=5$.

\subsection{Plasma echo test case}

To study the influence of trigonometric weights in large time, we then consider the plasma echo test case as proposed in [13]. The initial distribution function is given by:

$$
f(0, x, v)=\frac{1}{\sqrt{2 \pi}} e^{-v^{2} / 2}\left(1+\alpha \cos \left(k_{1} x\right)\right)
$$

with $\alpha=10^{-3}$ and $k_{1}=12 \pi / 100$. At time $t=200$, we add to the obtained particle distribution function, denoted $f\left(200^{-}, x, v\right)$, a second perturbation:

$$
f(200, x, v)=f\left(200^{-}, x, v\right)+\frac{1}{\sqrt{2 \pi}} e^{-v^{2} / 2} \alpha \cos \left(k_{2} x\right),
$$

where $k_{2}=25 \pi / 100$. The two waves are first damped and then their self-interactions results in echos, that are increase of the electrostatic energy.

Gauss versus trigonometric weights. On Figure 6, we plotted the time evolution of the electric energy (in log scale) computed with the SLDG scheme with Gauss and trigonometric weights. We compare the results with a referrenced solution obtain using a large number of points, $N_{x}=N_{v}=1024$, and a large degree of approximation $d=6$. We observe the two damping at time $t=0$ and time $t=100$, then the first interaction at time $t=270$ and the two echos at times $t=400$ and $t=800$.

We compare the solutions with $N_{x}=N_{v}=600$ and degrees $d=2,4$, and 6 . The expected recurrence time for the first perturbation equals $T_{\mathrm{rec}}=2 \pi /\left(k_{1} \Delta v\right)=625$ and it actually holds: the increase of electrostatic energy is significant for $d=2$ and less predominant for $d=4$ and 6 . Considering the trigonometric weights enables to reduce 

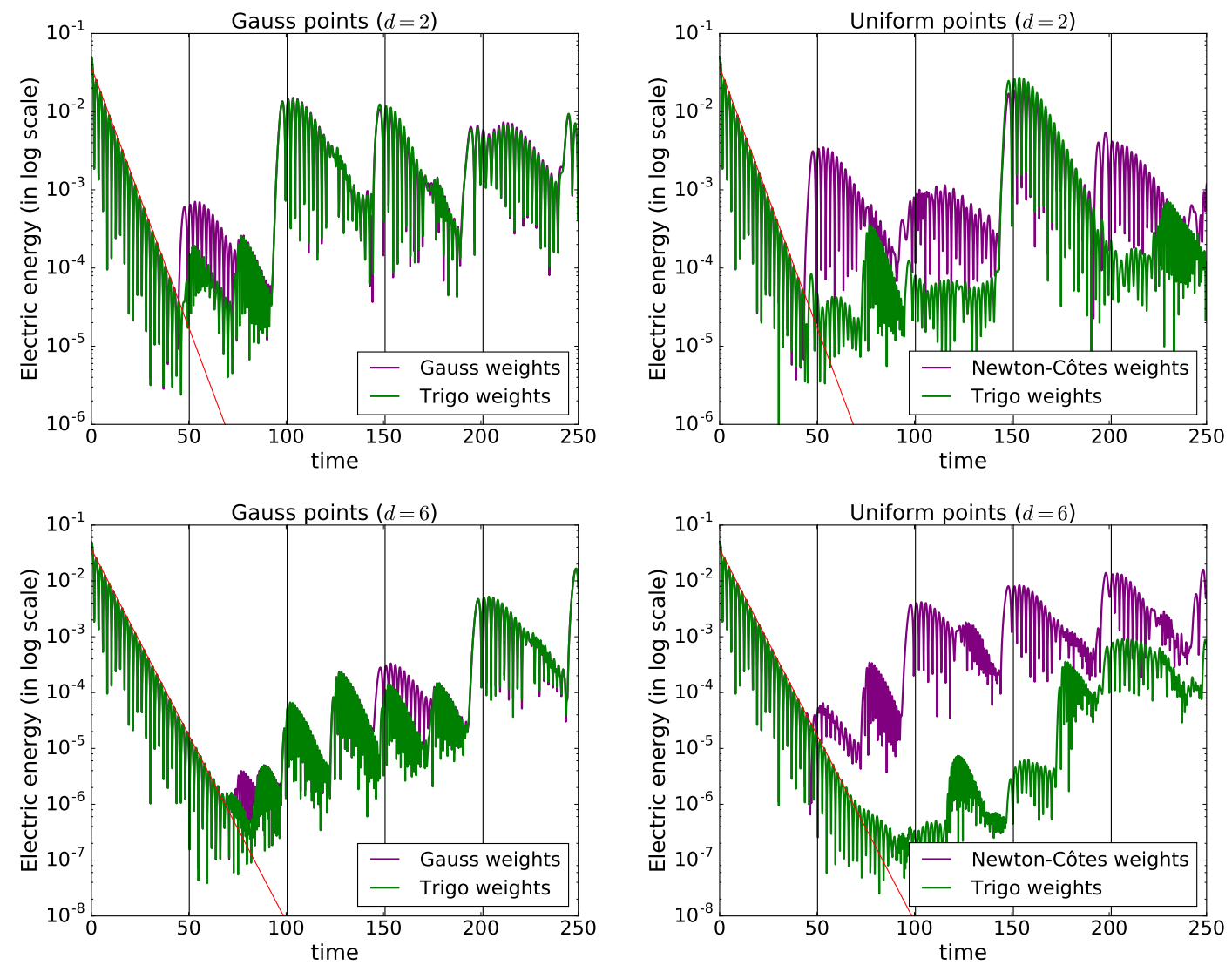

Figure 5: (Landau damping, SLDG, $k=0.5, \alpha=10^{-2}$ ) Time evolution of the electric energy $\mathcal{E}(t)$ (in log scale) when taking Gauss points (left) and uniform points (right). The black vertical lines indicates the multiple of the recurrence time $T_{\text {rec }}=2 \pi /(k \Delta v) \approx$ 50.26. Parameters: $N_{v}=40, v_{\max }=5$. 

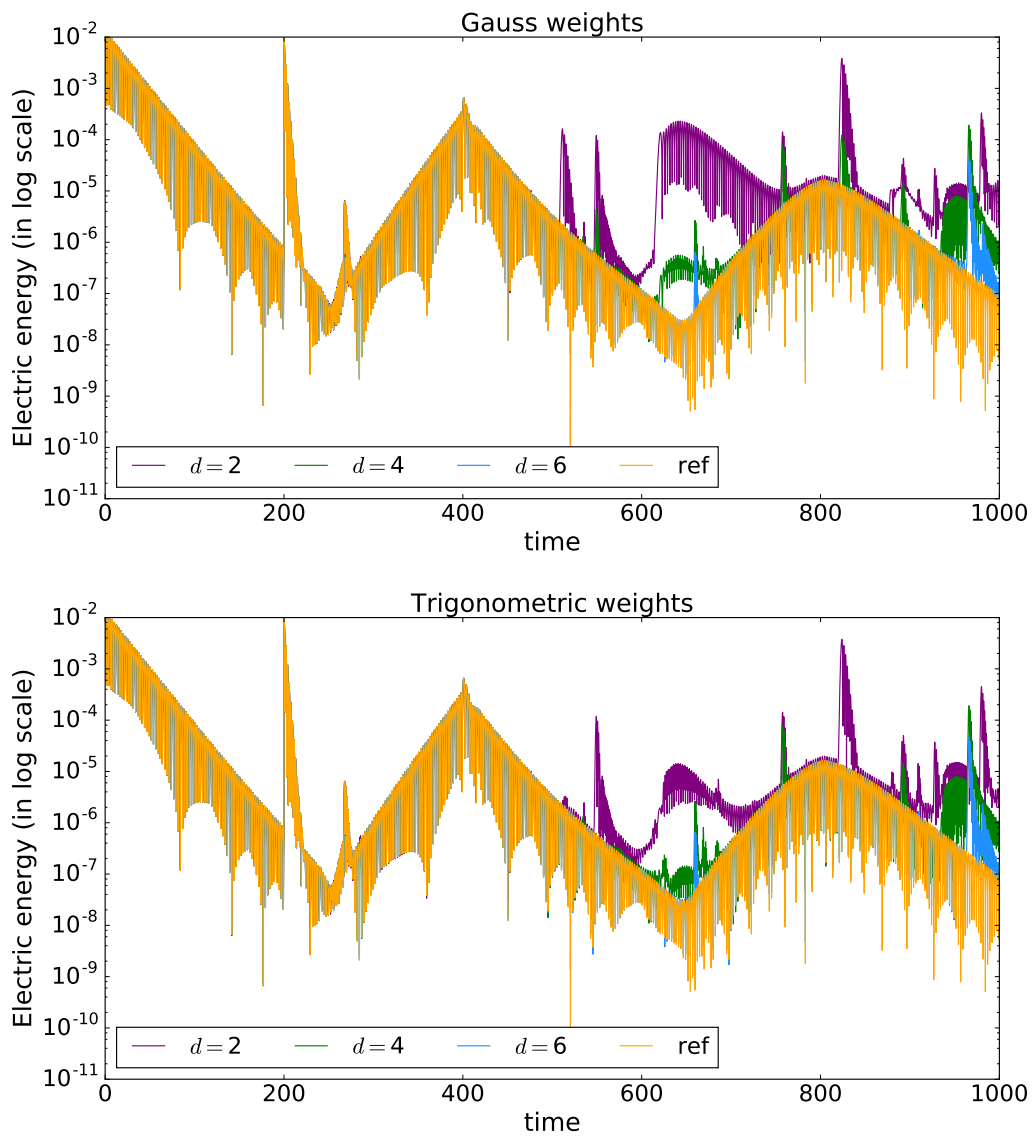

Figure 6: (Plasma echo, $\alpha=10^{-3}, k_{1}=12 \pi / 100, k_{2}=24 \pi / 100$ ) Time evolution of the electric energy $\mathcal{E}(t)$ (in $\log$ scale) when varying the degree $d=2,4,6$ for Gauss weights (top) and trigonometric weights (bottom). Parameters: $N_{v}=600, v_{\max }=8$. Reference solution (in orange): $N_{x}=N_{v}=1024$ and $d=6$.

this increase of energy. However, due to the interactions between the two waves, we observe that recurrence effect also occurs before, at time $t=510$ and $t=550$. Using trigonometric weights almost suppress the first peak of energy but seems to have no effect on the second one.

Gauss versus uniform points. In Figure 7, we compare the results when considering uniform points in the SLDG method, like in the previous Landau damping test case. We first note that, for the standard Gauss points and the degree $d=6$, the use of trigonometric points slightly improve the results just before the recurrence effect at time $t=965$. We then observe that considering uniform points, with standard weights (Newton-Côtes weights), results in the reappearance of a large recurrence effect at time $T_{\text {rec }}=2 \pi /\left(k_{1} \Delta v\right)=625$. This is partially corrected thanks to the trigonometric weights 

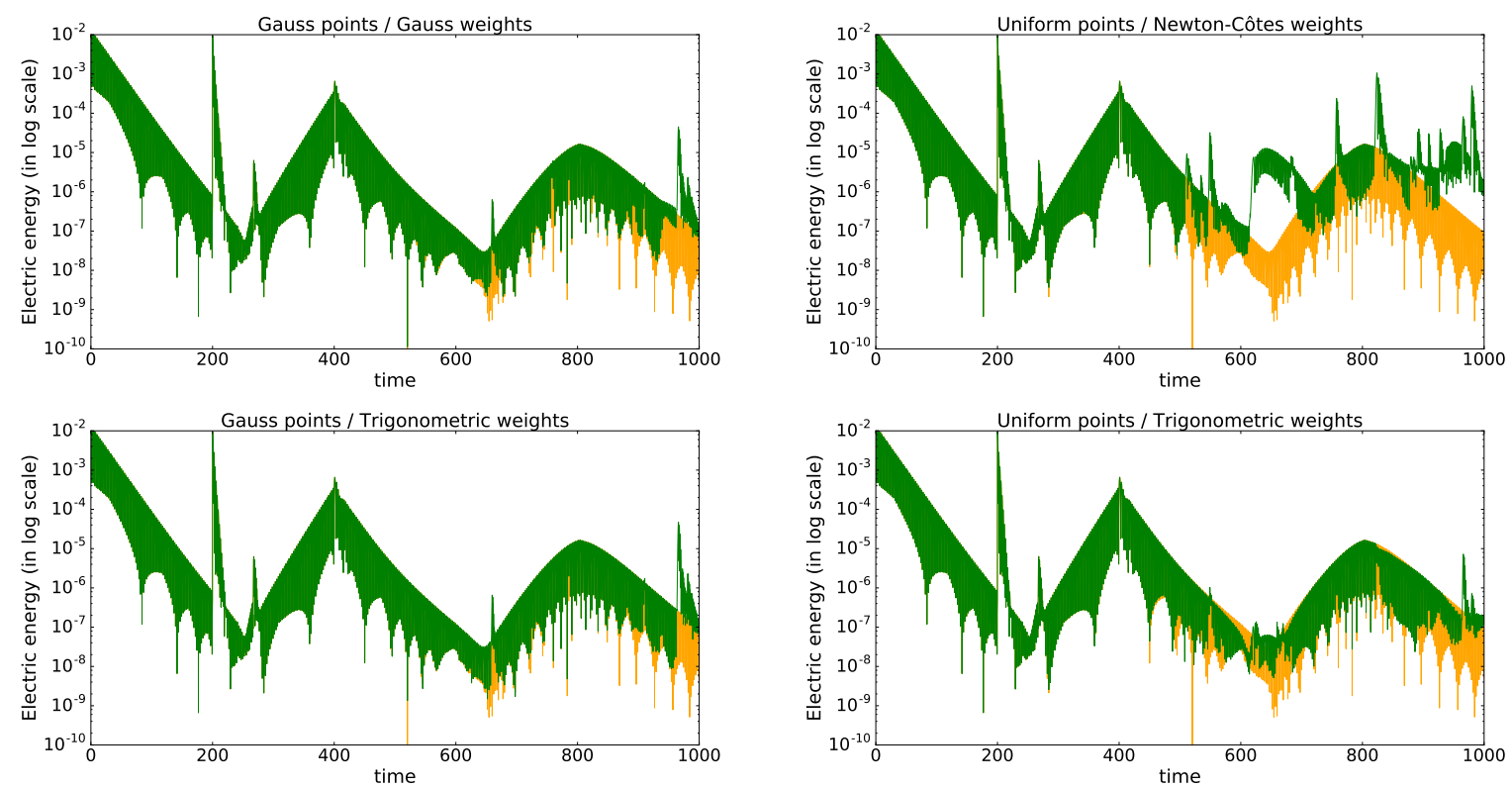

Figure 7: (Plasma echo, SLDG, $\alpha=10^{-3}, k_{1}=12 \pi / 100, k_{2}=24 \pi / 100$.) Time evolution of the electric energy $\mathcal{E}(t)$ (in log scale) for Gauss (left) / uniform (right) points and classical (top) / trigonometric (bottom) weights. Parameters: $N_{x}=N_{v}=600$, $v_{\max }=8, d=6$. Reference solution (in orange): $N_{x}=N_{v}=1024$ and $d=6$.

(bottom right figure), while still being less accurate than the version with Gauss points.

\section{Conclusion}

In this article, we have shown that the recurrence time for methods based on a regular finite-element mesh in velocity is related to the numerical quadrature of trigonometric polynomials. This result is obtained for the free-transport equation. Up to our knowledge, this link was not yet established. As a consequence, we also propose to use the trigonometric weights to shift in time the first occurence of the recurrence effect. On two test cases (Landau damping, plasma echo) and using two different solvers (SLDG, FESL), we show that the previous results extend to Vlasov-Poisson solvers at least in the linear regime. We also observe that the use of trigonometric weights is mostly efficient for low degree of approximation $d=2$ or 3 . Finally, we also show that using trigonometric weights is crucial when considering uniform degrees of freedom.

The main purpose of this work is to provide a validation tool for the concerned codes: it indeed indicates that the recurrence time always equals $T_{\text {rec }}=2 \pi /(k \Delta v)$ although the amplitude may be very small for large degree of approximation. It can also lead to further developments by combining the use of trigonometric weights to filtering techniques. 


\section{A Trigonometric quadrature with symmetric points}

We consider symmetric points $\left(\bar{v}_{\ell}\right)_{0 \leqslant \leqslant d}$ of the interval $[0,1]$. A quadrature rule with symmetric points and weights integrates exactly sine functions $\sin (2 \pi m x)$, with $m \in \mathbb{N}^{*}$ (as imparity). Therefore, it remains to demand that the quadrature rule integrates exactly cosine functions, which are even.

We can restrict the problem to integrate on the domain to $[0,0.5]$, where lie $\lfloor d / 2\rfloor+1$ quadrature points. We thus consider the following space:

$$
\mathcal{C}_{d}=\operatorname{Vect}\{\cos (2 \pi m x), \quad m \in \llbracket 0,\lfloor d / 2\rfloor \rrbracket\}
$$

which has dimension $\lfloor d / 2\rfloor+1$. Let $f(v):[0,0.5] \rightarrow \mathbb{R}$, find the trigonometric interpolant (in the cosine basis) $t \in \mathcal{C}_{d}$ such that $f\left(\bar{v}_{\ell}\right)=t\left(\bar{v}_{\ell}\right)$ for all $\ell \in \llbracket 0,\lfloor d / 2\rfloor \rrbracket$. This is equivalent to find the polynomial interpolant $p \in \mathbb{P}_{\lfloor d / 2\rfloor}$ such that: $f\left(\bar{v}_{\ell}\right)=p\left(\cos \left(2 \pi \bar{v}_{\ell}\right)\right)$ for all $\ell \in \llbracket 0,\lfloor d / 2\rfloor \rrbracket$. The trigonometric interpolant has the following expression:

$$
t(v)=p(\cos (2 \pi v))=\sum_{\ell=0}^{\lfloor d / 2\rfloor} f\left(v_{\ell}\right) L_{\ell}(\cos (2 \pi v)),
$$

where the $\left(L_{\ell}\right)_{\ell}$ are the elementary Lagrange polynomials:

$$
L_{\ell}(z)=\prod_{\substack{0 \leqslant j \leqslant\lfloor d / 2\rfloor \\ j \neq \ell}} \frac{z-\cos \left(2 \pi \bar{v}_{j}\right)}{\cos \left(2 \pi \bar{v}_{\ell}\right)-\cos \left(2 \pi \bar{v}_{j}\right)} .
$$

For any function $f(v):[0,0.5] \rightarrow \mathbb{R}$, we thus defined the quadrature rule as follows:

$$
\tilde{Q}(f)=\sum_{\ell=0}^{\lfloor d / 2\rfloor} \tilde{\omega}_{\ell} f\left(v_{\ell}\right),
$$

with weights given by:

$$
\forall \ell \in \llbracket 0,\lfloor d / 2\rfloor \rrbracket, \quad \tilde{\omega}_{\ell}=\int_{0}^{0.5} L_{\ell}(\cos (2 \pi v)) d v=\frac{1}{2} \int_{0}^{1} L_{\ell}(\cos (2 \pi v)) d v,
$$

which is exact on $\mathcal{C}_{d}$.

We then extend the formula on the interval $[0,1]$, using the parity of the cosine functions. For any function $f:[0,1] \rightarrow \mathbb{R}$, we consider the following quadrature rule:

$$
Q(f)=\sum_{\ell=0}^{d} \omega_{\ell} f\left(v_{\ell}\right)
$$

where the weights are defined by:

$$
\forall \ell \in \llbracket 0, d \rrbracket, \quad \omega_{\ell}=\left\{\begin{array}{ll}
\tilde{\omega}_{\ell}, & \text { if } \ell<d / 2 \\
2 \tilde{\omega}_{\ell}, & \text { if } \ell=d / 2 \\
\tilde{\omega}_{\ell-d} & \text { if } \ell>d / 2 .
\end{array} \quad \text { (even } d\right)
$$

By construction, this formula is exact on $\mathcal{C}_{d}$. The points and weights being symmetric, the formula is also exact on sine functions. The formula is thus exact on trigonometric polynomials of degree less or equal to $\lfloor d / 2\rfloor$. 


\section{B Semi-Lagrangian Discontinuous Galerkin (SLDG) method}

For the sake of completeness, we present here the semi-Lagrangian discontinuous Galerkin method (SLDG) (see also references [10]).

One-dimensional advection equation. We first present the method on the onedimensional advection equation. Let $g: \Omega_{v} \rightarrow \mathbb{R}$ satisfying the following equation:

$$
\partial_{t} g+a \partial_{v} g=0
$$

with $a \in \mathbb{R}$. We consider the finite-element mesh introduced in section 2.1 using the Gauss-Legendre points $\left(d+1\right.$ Gauss-Legendre points in each $N_{v}$ cell).

Let $\Delta t>0$ and $g^{n}(v)$ the interpolation solution at time $t^{n}=n \Delta t$ on the piecewise polynomial space

$$
\mathcal{V}=\left\{\psi \in L^{2}\left(\Omega_{v}\right) \mid \quad \psi_{\left[v_{j}, v_{j+1}\right]} \in \mathbb{P}_{d}, \forall j \in \llbracket-N_{v} / 2, N_{v} / 2-1 \rrbracket\right\} .
$$

We recall its expression:

$$
g^{n}(v)=\sum_{(j, \ell) \in K} g_{(j, \ell)}^{n} \phi_{(j, \ell)}(v),
$$

where $\phi_{j, \ell}(v)$ denotes the piecewise continuous and polynomial function, which is polynomial function of degree $\leqslant d$ on each cell $\left[v_{i}, v_{i+1}\right]$ and satisfies $\phi_{j, \ell}\left(v_{k, i}\right)=\delta_{(j, \ell),(i, k)}$, for $(j, \ell),(i, k) \in K$. The $\left(\phi_{(j, \ell)}\right)$ are orthogonal:

$$
\int_{\Omega_{v}} \phi_{(j, \ell}(v) \phi_{(\bar{j}, \bar{\ell})}(v) d v=\sum_{\left(j^{\prime}, \ell^{\prime}\right) \in K} \Delta v \omega_{\ell^{\prime}} \phi_{(j, \ell)}\left(v_{\left(j^{\prime}, \ell^{\prime}\right)}\right) \phi_{(\bar{j}, \bar{\ell})}\left(v_{\left(j^{\prime}, \ell^{\prime}\right)}\right)=\Delta v \omega_{\ell} \delta_{(j, \ell),\left(j^{\prime}, \ell^{\prime}\right)},
$$

and we have:

$$
\begin{aligned}
\frac{1}{\Delta v \omega_{\ell}} \int_{\Omega_{v}} g^{n}(v) \phi_{(j, \ell)}(v) d v & =\frac{g_{(j, \ell)}^{n}}{\Delta v \omega_{\ell}} \int_{\Omega_{v}} \phi_{(j, \ell)}(v)^{2} d v \\
& =\frac{g_{(j, \ell)}^{n}}{\Delta v \omega_{\ell}} \sum_{\left(j^{\prime}, \ell^{\prime}\right) \in K} \Delta v \omega_{\ell^{\prime}} \phi_{(j, \ell)}^{2}\left(v_{\left(j^{\prime}, \ell^{\prime}\right)}\right) \\
& =g_{(j, \ell)}^{n},
\end{aligned}
$$

where we use the fact that the Gauss-Legendre integration is exact for polynomials of degree lower or equal to $2 d+1$. Expression (12) is then an orthogonal decomposition with coefficients that coincide with nodal values (up to multiplicative factors).

To compute the approximate solution at time $t^{n+1}$, the semi-Lagrangian discontinuous Galerkin method (SLDG) consists in first advecting exactly the approximate solution and then taking the $L^{2}$ projection on $\mathcal{V}_{N_{v}, d}$ :

$$
g^{n+1}(v)=P_{\mathcal{V}}\left[\sum_{(j, \ell) \in K} g_{(j, \ell)}^{n} \phi_{(j, \ell)}(v-a \Delta t)\right],
$$


where $P_{\mathcal{V}}$ denotes the $L^{2}$ projection on $\mathcal{V}$. Consequently, the solution is given by:

$$
g^{n+1}(v)=\sum_{(j, \ell) \in K} g_{(j, \ell)}^{n+1} \phi_{(j, \ell)}(v)
$$

with coefficient given by:

$$
\begin{aligned}
g_{(j, \ell)}^{n+1} & =\frac{1}{\Delta v \omega_{\ell}} \int_{\Omega_{v}}\left[\sum_{\left(j^{\prime}, \ell^{\prime}\right) \in K} g_{\left(j^{\prime}, \ell^{\prime}\right)}^{n} \phi_{\left(j^{\prime}, \ell^{\prime}\right)}(v-a \Delta t)\right] \phi_{(j, \ell)}(v) d v \\
& =\frac{1}{\Delta v \omega_{\ell}} \sum_{\left(j^{\prime}, \ell^{\prime}\right) \in K}\left(\int_{\Omega_{v}} \phi_{\left(j^{\prime}, \ell^{\prime}\right)}(v-a \Delta t) \phi_{(j, \ell)}(v) d v\right) g_{\left(j^{\prime}, \ell^{\prime}\right)}^{n} .
\end{aligned}
$$

We denote this time step by:

$$
g^{n+1}=\mathcal{S}_{a \Delta t} g^{n}
$$

Remark 1. Due to the compact support of polynomials $\phi_{(j, \ell)}$, expression (14) actually involves only $2(d+1)$ non-zero terms. Denoting $\alpha=\lfloor-a \Delta t / \Delta v\rfloor$ the shift, we have:

$$
\begin{aligned}
g_{(j, \ell)}^{n+1}=\frac{1}{\Delta v \omega_{\ell}} & {\left[\sum_{\ell^{\prime} \in K}\left(\int_{\Omega_{v}} \phi_{\left(j+\alpha, \ell^{\prime}\right)}(v-a \Delta t) \phi_{(j, \ell)}(v) d v\right) g_{\left(j+\alpha, \ell^{\prime}\right)}^{n}\right.} \\
& \left.+\sum_{\ell^{\prime} \in K}\left(\int_{\Omega_{v}} \phi_{\left(j+\alpha+1, \ell^{\prime}\right)}(v-a \Delta t) \phi_{(j, \ell)}(v) d v\right) g_{\left(j+\alpha+1, \ell^{\prime}\right)}^{n}\right]
\end{aligned}
$$

This could be written in matrix formulation:

$$
\begin{aligned}
\forall j, \quad g_{(j, .)}^{n+1} & =\frac{1}{\Delta v \omega_{\ell}}\left[A(\alpha) g_{(j, .)}^{n}+B(\alpha) g_{(j, .)}^{n}\right], \\
A(\alpha) & =\left(\int_{\Omega_{v}} \phi_{\left(1+\alpha, \ell^{\prime}\right)}(v-a \Delta t) \phi_{(1, \ell)}(v) d v\right)_{\ell, \ell^{\prime} \in I_{d}} \in M_{d+1}(\mathbb{R}), \\
B(\alpha) & =\left(\int_{\Omega_{v}} \phi_{\left(1+\alpha+1, \ell^{\prime}\right)}(v-a \Delta t) \phi_{(1, \ell)}(v) d v\right)_{\ell, \ell^{\prime} \in I_{d}} \in M_{d+1}(\mathbb{R}) .
\end{aligned}
$$

Application to the Vlasov equation. We now briefly present how the SLDG scheme combined to splitting method can be used to solve the Vlasov-Poisson system.

We consider the same finite-element mesh for the velocity domain $(d+1$ GaussLegendre points in each $N_{v}$ cell). The spatial mesh is constructed similarly: let $N_{x} \in \mathbb{N}$, $\Delta x=\left(x_{\max }-x_{\min }\right) / N_{x}$ and the finite-element nodes: $x_{j, m}=x_{\min }+\left(k-1+\bar{x}_{m}\right) \Delta x / 2$ for all $k \in \llbracket 1, N_{x} \rrbracket, m \in \llbracket 0, d \rrbracket$, where the $\left(\bar{x}_{m}\right)$ also denotes the $d+1$ Gauss-Legendre points in $[-1,1]$. We denote by $f_{(k, m),(j, \ell)}^{n}$ the approximate value of the distribution function $f$ at point $\left(x_{(k, m)}, v_{(j, \ell)}\right)$ at time $t_{n}=n \Delta t$.

The $n$-th iteration of the scheme writes: 
1. Advection in space. We solve the $2 N_{v}(d+1)$ transport equations on time interval $\Delta t$ :

$$
\forall(j, \ell) \in \llbracket-N_{v}, N_{v} \rrbracket^{*} \times I_{d}, \quad \partial_{t} f_{.,(j, \ell)}(x, t)+v_{(j, \ell)} \partial_{x} f_{.,(j, \ell)}(x, t)=0,
$$

using the SLDG scheme:

$$
f_{.,(j, \ell)}^{*}=\mathcal{S}_{v_{(j, \ell)} \Delta t} f_{.,(j, \ell)}^{n}
$$

where $f_{.,(j, \ell)}$ denotes the distribution function at velocity $v=v_{j, \ell}$.

2. Compute the charge density $\rho_{(k, m)}^{*}$, corresponding to $f^{*}$, and then the electric field $E_{(k, m)}^{*}$ from the Poisson equation $-\partial_{x} E^{*}=\overline{\rho^{*}}-\rho^{*}$ with a finite difference or a FFT solver (where $\overline{\rho^{*}}$ denotes the average of $\rho^{*}$ over $\Omega_{x}$ ).

3. Advection in velocity. We solve the $N_{x}(d+1)$ transport equations on time interval $\Delta t$ :

$$
\forall(k, m) \in \llbracket 1, N_{x} \rrbracket \times I_{d}, \quad \partial_{t} f_{(k, m), . .}(v, t)+E_{(k, m)}^{*} \partial_{v} f_{(k, m), .}(v, t)=0,
$$

using the SLDG scheme:

$$
f_{(k, m), .}^{n+1}=\mathcal{S}_{E_{(k, m)}^{*} \Delta t} f_{(k, m), .}^{*},
$$

where $f_{(k, m)}$, denotes the distribution function at position $x=x_{k, m}$.

In this third step, the computation of the charge density can be made using any quadrature rules. In particular, we can use the Gauss or the trigonometric rules. In the first and second step, the advected piece-wise polynomials can be expessed in any basis and thus we can use a Lagrange polynomial basis based on uniform points instead of Gauss-Legendre points.

We here present a first order splitting. Higer oder splitting could be also considered. In practice, we will use the second-order Strang splitting (see [?]).

\section{Finite Element/Semi-Lagrangian (FE/SL) method}

We here briefly present the Finite Volume/Finite element method introduced in [18,20, $25]$.

We consider the finite-element mesh introduced in section 2.1 with Gauss-Lobatto points. Since we will consider continuous interpolation, we introduce the set $K^{\prime}=$ $\llbracket-N_{v} / 2, N_{v} / 2-1 \rrbracket \times \llbracket 0, d-1 \rrbracket \cup\left\{\left(N_{v} / 2-1, d\right)\right\}$. Let $\left(\varphi_{(j, \ell)}\right)_{(j, \ell) \in K^{\prime}}$ a nodal basis of the following continuous finite-element space

$$
\mathcal{V}^{c}=\left\{\psi \in L^{2}\left(\Omega_{v}\right) \cap C\left(\Omega_{v}\right) \mid \quad \psi_{\mid\left[v_{j}, v_{j+1}\right]} \in \mathbb{P}_{d}, \quad \forall j \in \llbracket-N_{v} / 2, N_{v} / 2-1 \rrbracket\right\} .
$$

It satisfies the relation: $\varphi_{(j, \ell)}\left(v_{(i, n)}\right)=1$ if $v_{(j, \ell)}=v_{(i, n)}$ and 0 otherwise. We then expand the distribution function in velocity:

$$
f(x, v, t)=\sum_{(j, \ell) \in K^{\prime}} w_{(j, \ell)}(x, t) \varphi_{(j, \ell)}(v)
$$


where $w(x, t)=\left(w_{(j, \ell)}(x, t)\right)_{(j, \ell) \in K^{\prime}}$ denotes the set of $N_{v} d+1$ components.

The method is based on the weak-formulation:

$$
\partial_{t} \int_{\Omega_{v}} f \varphi+\partial_{x} \int_{\Omega_{v}} v f \varphi+\int_{\Omega_{v}} E \partial_{v} f \varphi-\frac{1}{2} \int_{\partial \Omega_{v}}\left(E \cdot n_{v}\right)^{-} f \varphi=0, \quad \forall \varphi \in \mathcal{V}^{c},
$$

is then equivalent to

$$
\begin{aligned}
& \forall(i, n) \in K^{\prime} \\
& \sum_{(j, \ell) \in K^{\prime}}\left(\partial_{t} w_{(j, \ell)} \int_{\Omega_{v}} \varphi_{(j, \ell)} \varphi_{(i, n)}+\partial_{x} w_{(j, \ell)} \int_{\Omega_{v}} v \varphi_{(j, \ell)} \varphi_{(i, n)}+w_{(j, \ell)} \int_{\Omega_{v}} \varphi_{(i, n)}\left(E \partial_{v}\right) \varphi_{(j, \ell)}\right. \\
& \left.\quad-\frac{1}{2} w_{(j, \ell)}\left(E^{-} \varphi_{(j, \ell)}\left(v_{\max }\right) \varphi_{(i, n)}\left(v_{\max }\right)-E^{+} \varphi_{(j, \ell)}\left(-v_{\max }\right) \varphi_{(i, n)}\left(-v_{\max }\right)\right)\right)=0,
\end{aligned}
$$

The matrix formulation of these equations, referred as the reduced Vlasov equation, writes

$$
M \partial_{t} w+A \partial_{x} w+B(E) w=0 .
$$

The mass matrix $M$ and the matrices $A, B(E)$ are matrices of dimension $N_{v} d+1$, whose elements (indexed by $K^{\prime} \times K^{\prime}$ ) are given by

$$
\begin{aligned}
M_{(i, n),(j, \ell)}= & \int_{\Omega_{v}} \varphi_{(i, n)} \varphi_{(j, \ell)}, \quad A_{(i, n),(j, \ell)}=\int_{\Omega_{v}} v \varphi_{(i, n)} \varphi_{(j, \ell)}, \\
B(E)_{(i, n),(j, \ell)}= & \int_{\Omega_{v}} \varphi_{(i, n)}\left(E \partial_{v}\right) \varphi_{(j, \ell)} \\
& +\frac{1}{2} E^{+} \varphi_{(j, \ell)}\left(-v_{\max }\right) \varphi_{(i, n)}\left(-v_{\max }\right)-\frac{1}{2} E^{-} \varphi_{(j, \ell)}\left(v_{\max }\right) \varphi_{(i, n)}\left(v_{\max }\right) .
\end{aligned}
$$

When the integrals appearing in the definion of matrices $M, A$ and $B(E)$ are computed using Gauss-Lobatto quadrature, then matrices $M$ and $A$ are diagonal: this is the mass lumping technique. We thus end up with a system of $N_{v} d+1$ coupled transport equations. The first order splitting is thus given by:

1. Advection in space. We solve the $N_{v} d+1$ transport equation on time interval $\Delta t:$

$$
\forall(i, n) \in K^{\prime}, \quad \partial_{t} w_{(k, \ell)}+\left(M^{-1} A\right)_{(k, \ell),(k, \ell)} \partial_{x} w_{(k, \ell)}=0
$$

We here propose to solve them on a regular mesh in space using a semi-Lagrangian scheme with a local Lagrange interpolation [9]. We thus obtain $w^{*}$ from $w^{n}$.

2. Compute the charge density $\rho^{*}$, corresponding to $w^{*}$, and the electric field $E^{*}$ from the Poisson equation $-\partial_{x} E^{*}=\overline{\rho^{*}}-\rho^{*}$ with a finite difference or a FFT solver (where $\overline{\rho^{*}}$ denotes the average of $\rho^{*}$ over $\Omega_{x}$ ).

3. Advection in velocity. We solve on time interval $\Delta t$ the system of differential equations:

$$
\partial_{t} w=M^{-1} B\left(E^{*}\right) w
$$

using the Euler scheme.

Second-order scheme in time can also be considered. We refer to $[18,25]$ for more details. 


\section{References}

[1] Abbasi, H. and Jenab, M.H. and Pajouh, H. Hakimi. Preventing the recurrence effect in the Vlasov simulation by randomizing phase-point velocities in phase space. Phys. Rev. E, 84(3):036702, 2011.

[2] A.P. Austin. Some new results on and applications of interpolation in numerical computation. PhD thesis, Mathematical Institute, University of Oxford, Oxford, UK, 2016.

[3] A.P. Austin and L.N. Trefethen. Trigonometric interpolation and quadrature in perturbed points. SIAM J. Numer. Anal., 55(5):2113-2122, 2017.

[4] N. Ayuso, J.A. Carrillo, and C.-H. Shu. Discontinuous Galerkin methods for the one-dimensional Vlasov-Poisson system. Kinet. Relat. Models, 4(4):955-989, 2011.

[5] B. Ayuso De Dios, J. A. Carrillo, and C.-W. Shu. Discontinuous Galerkin methods for the multi-dimensional Vlasov-Poisson problem. Math. Models Methods Appl. Sci., 22(12):1250042, 2012.

[6] Z. Cai and Y. Wang. Suppression of recurrence in the Hermite-spectral method for transport equations. arXiv preprint arXiv:1709.08194, 2017.

[7] C.-Z. Cheng and G. Knorr. The integration of the Vlasov equation in configuration space. J. Comput. Phys., 22(3):330-351, 1976.

[8] Y. Cheng, I.M. Gamba, and P.J. Morrison. Study of conservation and recurrence of Runge-Kutta discontinuous Galerkin schemes for Vlasov-Poisson systems. J. Sci. Comput, 56(2):319-349, 2013.

[9] N. Crouseilles, M. Mehrenberger, and E. Sonnendrücker. Conservative semilagrangian schemes for vlasov equations. J. Comput. Phys., 229(6):1927-1953, 2010.

[10] N. Crouseilles, M. Mehrenberger, and F. Vecil. Discontinuous Galerkin semilagrangian method for Vlasov-Poisson. ESAIM: Proc., 32:211-230, 2011.

[11] L. Einkemmer. A study on conserving invariants of the Vlasov equation in semiLagrangian computer simulations. J. Plasma Phys., 83(2), 2017.

[12] L. Einkemmer and A. Ostermann. Convergence analysis of a discontinuous Galerkin/Strang splitting approximation for the Vlasov-Poisson equations. SIAM J. Numer. Anal., 52(2):757-778, 2014.

[13] L. Einkemmer and A. Ostermann. A strategy to suppress recurrence in grid-based Vlasov solvers. Eur. Phys. J. D., 68:197, 2014.

[14] Bengt Eliasson. Outflow boundary conditions for the Fourier transformed onedimensional Vlasov-Poisson system. Journal of Scientific Computing, 16(1):1-28, 2001. 
[15] Bengt Eliasson. Numerical modelling of the two-dimensional Fourier transformed Vlasov-Maxwell system. J. Comput. Phys., 190(2):501-522, 2003.

[16] Bengt Eliasson. Numerical simulations of the Fourier-transformed Vlasov- Maxwell system in higher dimensions - Theory and applications. Transport Theory and Statistical Physics, 39:387-465, 2010.

[17] F. Filbet, E. Sonnendrücker, and P. Bertrand. Conservative numerical schemes for the vlasov equation. J. Comput. Phys., 172:166-187, 2001.

[18] S. Guisset, P. Helluy, M. Massaro, L. Navoret, N. Pham, and M. Roberts. Lagrangian/Eulerian solvers and simulations for Vlasov-Poisson. ESAIM: Proc., 53:120-132, 2016.

[19] R. E. Heath, I.M. Gamba, P.J. Morrison, and C. Michler. A discontinuous Galerkin method for the Vlasov-Poisson system. J. Comput. Phys., 231(4):1140-1174, 2012.

[20] P. Helluy, N. Pham, and A. Crestetto. Space-only hyperbolic approximation of the Vlasov equation. ESAIM: Proc., 43:17-36, 2013.

[21] R.W. Hockney and J.W. Eastwood. Computer simulation using particles. CRC Press, 1988.

[22] A.J. Klimas and W.M. Farrell. A splitting algorithm for Vlasov simulation with filamentation filtration. J. Comput. Phys., 110(1):150-163, 194.

[23] E. Madaule, M. Restelli, and Eric Sonnendrücker. Energy conserving discontinuous Galerkin spectral element method for the Vlasov-Poisson system. J. Comput. Phys., 279:261-288, 2014.

[24] F. Peherstorfer. Positive trigonometric quadrature formulas and quadrature on the unit circle. Math. Comp., 80, 2011.

[25] N. Pham. Numerical methods for the reduced Vlasov equation. PhD thesis, Université de strasbourg, 2016.

[26] J.-M. Qiu and C.-W. Shu. Positivity preserving semi-Lagrangian discontinuous Galerkin formulation: theoretical analysis and application to the Vlasov-Poisson system. J. Comput. Phys., 230(23):8386-8409, 2011.

[27] J.A. Rossmanith and D.C. Seal. A positivity-preserving high-order semi-Lagrangian discontinuous Galerkin scheme for the Vlasov-Poisson equations. J. Comput. Phys., 230(16):6203-6232, 2011.

[28] Eric Sonnendrücker. Numerical methods for the Vlasov equations. In preparation. 